Hautarzt 2021 · 72:44-49

https://doi.org/10.1007/s00105-020-04726-9

Online publiziert: 26 . November 2020

(c) Der/die Autor(en) 2020

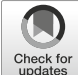

\section{Zusatzmaterial online}

Die Online-Version dieses Beitrags (https:// doi.org/10.1007/s00105-020-04726-9) enthält zusätzlich 12 Abbildungen. Beitrag und Zusatzmaterial stehen Ihnen im elektronischen Volltextarchiv auf https:// www.springermedizin.de/der-hautarzt zur Verfügung. Sie finden das Zusatzmaterial am Beitragsende unter "Supplementary Material".

Grundsätzlich werden in der Pharmakologie pharmakodynamische und -kinetische Wechselwirkungen unterschieden. Dabei stellt die Pharmakodynamik die Analyse der biologischen Wirkung eines Pharmakons dar und beschreibt den Einfluss von Arzneimitteln auf den Organismus.

Pharmakokinetische Interaktionen beziehen sich auf Prozesse, die ein Arzneimittel im Körper durchläuft (OnlineAbb. 1).

Das Risiko von Medikamenteninteraktionen steigt nicht nur durch die Polypharmazie, sondern auch durch Funktionsstörungen wichtiger Organe wie Leber, Nieren und Herzfunktion sowie durch eingeschränkte Stoffwechselleistungen bei Adipositas, Hypothyreose und Hypoproteinämie [1-4]. Genetische Faktoren können ebenfalls die Metabolisierung von Medikamenten beeinflussen (Online-Abb. 2). So kommt es beispielsweise durch einen angeborenen Mangel des Enzyms Thiopurine-Methyltransferase (TPMT) zur Akkumulation von Abbauprodukten von Azathioprin und damit zu vermehrten Nebenwirkungen.

Die Abbildungen "Online-Abb. 1-12" sind aus Platzgründen nur elektronisch als ESM verfügbar.

Kristina Krause - Katharina Jahn - Bernhard Homey

Klinik für Dermatologie, Universitätsklinikum Düsseldorf, Düsseldorf, Deutschland

\title{
Wechselwirkungen in der dermatologischen Systemtherapie
}

Durch den genetischen Polymorphismus am NAT2-Lokus kommt es zur Ausprägung eines langsamen und eines schnellen Acetylator-Phänotyps der Arylamin-N-Acetyltransferase 2, die unter anderem Medikamente wie Isoniazid, Hydralazin und Dapson verstoffwechselt. So geht eine reduzierte Metabolisierung von Isoniazid mit deutlich mehr hepatotoxischen Nebenwirkungen einher. Der genetische Polymorphismus des Enzyms CYP2D6, das am Stoffwechsel etwa jedes vierten Arzneimittels beteiligt ist, führt bei einer genetisch bedingten Defizienz zu einer deutlich verlangsamten Elimination des Arzneimittels aus dem Körper, was eine relative Überdosierung mit entsprechend verstärkten Nebenwirkungen bedingt [5]. Eine Bestimmung der TPMT-Aktivität ist im Rahmen einer Therapie mit Azathioprin unerlässlich.

Expertentipp. Um eine Verzögerung des Therapiebeginns mit Azathioprin bis zum Eingang der TPMT-Aktivität zu umgehen, empfehlen wir am Tag der Abnahme eine dosisreduzierte Einleitung von Azathioprin und eine ergebnisabhängige Anpassung im Verlauf.

Eine weitere genetische Prädisposition mit hoher Relevanz für den klinischen Alltag stellt der Glucose-6-Phosphat-Dehydrogenase-Mangel dar. Dabei handelt es sich um eine Mutation im x-chromosomalen G6PD-Gen, wodurch es zu einer erhöhten Anfälligkeit der Erythrozytenmembran für freie Sauerstoffradikale kommt. Bei erhöhtem oxidativem Stress, z.B. medikamentös bedingt durch eine Therapie mit Dapson, kann es bei Vorliegen eines Glucose-6-Phosphat-Dehydrogenase-Mangels zu einer Zerstörung der
Erythrozyten bis hin zur hämolytischen Krise kommen [16].

\section{Pharmakokinetische Interaktionen}

Die Pharmakokinetik beschreibt den Einfluss des Organismus auf die Arzneistoffe. Im Wesentlichen geht es um diejenigen Vorgänge, die nach der Gabe eines Medikamentes in den Verteilungsräumen des Körpers ablaufen. Hierzu zählen Resorption, Distribution, biochemische Um- und Abbauprozesse (Metabolisierung) sowie Elimination.

Bei der gleichzeitigen Verabreichung mehrerer Arzneimittel kann es zu einer gegenseitigen Beeinflussung der pharmakokinetischen Prozesse und einer Änderung konzentrationsabhängiger Wirkungen kommen.

\section{Lebermetabolismus}

Die meisten pharmakokinetischen Interaktionen beruhen auf einer veränderten Metabolisierung in der Leber durch Induktion oder Inhibition der Cytochrom-P450(CYP)-Isoenzyme. Mehr als die Hälfte dieser Reaktionen finden über das Isoenzym CYP 3A4 statt. Die häufigsten Induktoren, Inhibitoren und Substrate sind in 0 Abb. 1 zusammengefasst, wobei sowohl Inhibitoren als auch Induktoren ebenfalls als Substrate der CYP-Enzyme fungieren können. Hierbei ist insbesondere auf Medikamente mit einer geringen therapeutischen Breite $\mathrm{zu}$ achten, wie beispielsweise Ciclosporin, Digoxin, Phenytoin, Dapson und Warfarin, deren verringerte oder vermehrte Metabolisierung zu lebensbedrohlichen 


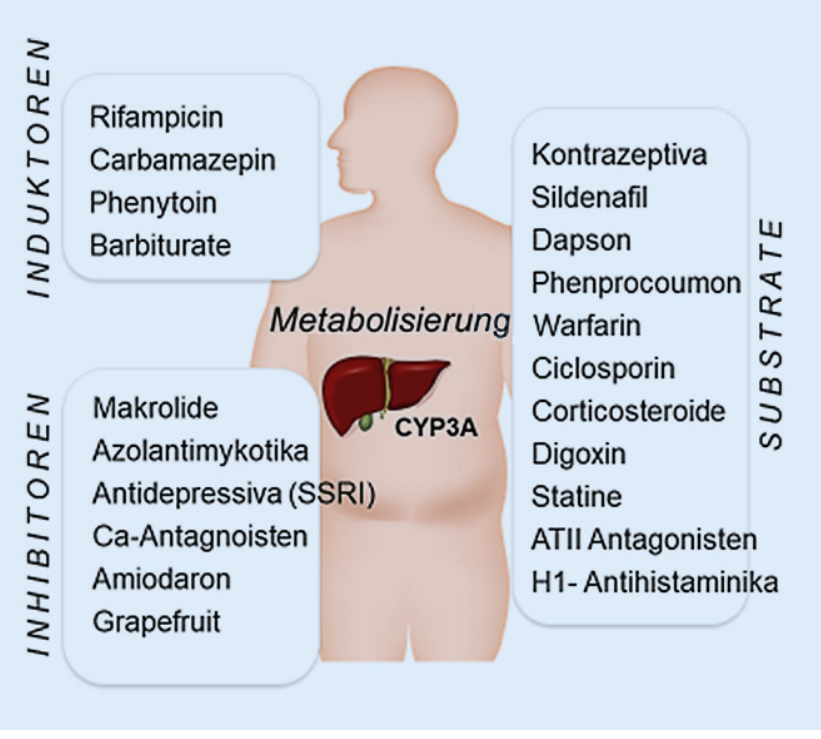

\section{Absorption}

Antibiotika wie beispielsweise Makrolide führen nicht nur über eine Inhibition der CYP-Metabolisierung zu einem erhöhten Plasmawirkspiegel der VitaminK-Antagonisten (Phenprocoumon), sondern steigern deren Wirksamkeit und das damit verbundene Blutungsrisiko auch durch eine Reduktion Vitamin-K-produzierender Darmbakterien und einer folglich verminderten Vitamin-K-Resorption (Online-Abb. 5).

\section{Renale Elimination}

Während bei den meisten Interaktionen eine genauere Quantifizierung des Ausmaßes der Interaktion nicht vorhersagbar ist, kann für renal eliminierte Pharmaka in Abhängigkeit von der glomerulären Filtrationsrate (GFR) eine Dosisadaptation berechnet werden. Dies muss insbesondere bei der Gabe von Aciclovir im Rahmen einer Zoster-Erkrankung bei älteren Patienten oder bei vorbekannter Niereninsuffizienz beachtet werden. Dabei sollte eine gleichzeitige Verabreichung nephrotoxischer Medikamente (Ciclosporin, Aminoglykoside, nichtsteroidale Antirheumatika, Penicilline und Cephalosporine) vermieden werden (Online-Abb. 6). Eine Alternative zur Behandlung des Zosters stellt das antivirale Arzneimittel Brivudin dar. Während Brivudin auch bei niereninsuffizienten Patienten angewendet werden kann, besteht für die Behandlung von immunsupprimierten Patienten aufgrund der unzureichenden Studienlage keine Zulassung. Kontraindiziert ist eine Therapie mit Brivudin bei gleichzeitiger Gabe von 5-Fluoruracil aufgrund einer gesteigerten Toxizität.

Auch bei der Verabreichung von Methotrexat muss sowohl vor als auch während der Therapie auf eine ausreichende Nierenfunktion geachtet werden. Bei bereits eingeschränkter Nierenfunktion sollte Methotrexat mit Vorsicht angewandt und ab einer GFR von $<60 \mathrm{ml} / \mathrm{min}$ auf die Hälfte der Dosis reduziert werden. Liegt eine stark reduzierte Nierenfunktion (GFR $<30 \mathrm{ml} / \mathrm{min}$ ) vor, darf Methotrexat nicht verabreicht werden [7]. 
Die Eliminierung von Methotrexat erfolgt renal über glomeruläre Filtration sowie aktive tubuläre Sekretion. Bei eingeschränkter Nierenfunktion und verminderter renaler Elimination kommt es zu einer verlängerten Plasmahalbwertszeit und einer gesteigerten intrazellulären Aufnahme und Akkumulation von Methotrexat [15]. Wird die intrazelluläre Aufnahmekapazität überschritten, kommt es zur Suppression der Replikation von schnell proliferierendem Gewebe. Myelodepression bis hin zu Agranulozytose sind mögliche und schwerwiegende Folgen.

Die Evaluation der GFR erfolgt im klinischen Routinealltag primär über den Kreatininwert im Serum. Dieser ist alters- und geschlechtsabhängig und steigt im Serum erst bei einer Reduktion der GFR auf etwa $50 \mathrm{ml} / \mathrm{min}$ (Normwert $130 \mathrm{ml} / \mathrm{min}$ ). Daraus ergibt sich, dass eine Einschränkung der GFR bis zu diesem Punkt nicht erkannt wird. Cystatin C hingegen wird frei glomerulär filtriert, im proximalen Tubulus komplett reabsorbiert und katabolisiert. Die Konzentration von Cystatin C im Serum wird somit weitestgehend durch die glomeruläre Filtrationsrate (GFR) bedingt und ermöglicht eine höhere diagnostische Genauigkeit.

Gerade bei der Verabreichung mehrerer Medikamente, die allein oder in Kombination Einfluss auf die renale Clearance nehmen können [8], sollte eine zusätzliche Bestimmung der GFR über die Cystatin-C-Serumkonzentration erfolgen. Gegebenenfalls ist eine Umstellung der Medikation von nierenfunktionsabhängigen auf nierenfunktionsunabhängige Medikamente zu empfehlen (• Tab. 1).

\section{Pharmakodynamische Interaktionen}

In der Pharmakodynamik, die das Wirkprofil eines Medikaments am Zielort beschreibt, können additive bzw. synergistische und antagonistische Wechselwirkungen von Medikamenten unterschieden werden. Ein synergistischer Effekt ist oftmals erwünscht und findet in Form von Kombinationstherapien unter anderem in der Schmerztherapie nach WHO(World Health Organiza-

Hautarzt 2021 · 72:44-49 https://doi.org/10.1007/s00105-020-04726-9

(c) Der/die Autor(en) 2020

\section{Wechselwirkungen in der dermatologischen Systemtherapie}

\section{Zusammenfassung}

Schwere Arzneimittelnebenwirkungen stellen mit 5-7\% einen häufigen Grund für eine Krankenhauseinweisung dar. Die Prävalenz von unerwünschten Arzneimittelwirkungen (UAW) während eines stationären Aufenthaltes liegt sogar bei etwa $11,5 \%$. Die Ursache sind oftmals Medikamentenwechselwirkungen, bedingt durch die Polypharmazie der multimorbiden älteren Patienten. So nimmt ein 65-jähriger Patient im Durchschnitt 5 Medikamente gleichzeitig ein. Aufgrund des zunehmenden Einsatzes von Systemtherapeutika in der Dermatologie und der gleichzeitig zunehmenden Polypharmazie ist das Wissen um Medikamenteninteraktionen für den Dermatologen zur Vermeidung schwerer Arzneimittelnebenwirkungen wesentlich. Dieser Beitrag soll eine Hilfestellung bieten, Patienten und Medikamente mit einem hohen Risiko für schwerwiegende Interaktionen zu identifizieren und dadurch das Auftreten unerwünschter Wirkungen oder auch die Verminderung des therapeutischen Effektes von Wirkstoffen zu vermeiden. Wir möchten darauf hinweisen, dass die folgende Arbeit einzelne Aspekte behandelt und nicht von der Überprüfung einzelner Arzneimittelinteraktionen mittels Interaktionsprogrammen entbindet. Nicht außer Acht gelassen werden sollte, dass neben verschreibungspflichtigen Medikamenten auch Nahrungsmittel, Nahrungsergänzungsmittel und Kräuter zu Interaktionen mit Medikamenten führen können.

\section{Schlüsselwörter}

Arzneimittelnebenwirkungen · Krankenhauseinweisung · Unerwünschte Arzneimittelwirkungen $\cdot$ Medikamentenwechselwirkungen · Polypharmazie

\section{Drug interactions in dermatological systemic treatment}

\section{Abstract}

Severe pharmacological side effects have an occurrence of $5-7 \%$ and represent a frequent reason for hospital admission. The prevalence of undesired pharmacological side effects during hospitalization is even higher with approximately $11.5 \%$. The causes are often interactions between drugs due to the polypharmacy of multimorbid older patients. On average, a 65 -year-old male patient will simultaneously be taking 5 medications. Due to the increasing use of systemic drugs in dermatology and the simultaneously increasing polypharmacy, knowledge of interactions between medications is essential for dermatologists in order to avoid severe side effects of drugs. This article provides assistance in order to identify patients and medications with a high risk for severe interactions and, therefore, to avoid the occurrence of undesired effects or the reduction of the therapeutic effects of active substances. We would like to point out that this article deals with individual aspects and does not mean that the testing of individual drug interactions with interaction programs can be omitted. It should also not be neglected that in addition to prescriptiononly drugs, foodstuffs, dietary supplements and herbs can also lead to interactions with medications.

\section{Keywords}

Drug side effects $\cdot$ Hospitalization $\cdot$ Adverse drug reactions - Drug interactions. Polypharmacy
tion)-Stufenschema, bei der Behandlung mit Antiinfektiva, in der Tumortherapie oder bei der Behandlung der Hypertonie Anwendung. Eine synergistische Wirkung kann jedoch auch unerwünschte Komplikationen hervorrufen. So besteht beispielsweise durch die gleichzeitige Gabe von Methotrexat und Sulfonamiden das erhöhte Risiko des Auftretens einer Panzytopenie infolge einer Knochen- marksuppression bedingt durch einen additiven Folsäureantagonismus [9].

\section{QT-Zeit-Verlängerung}

Sowohl pharmakodynamische als auch pharmakokinetische Arzneimittelinteraktionen können eine QT-Zeit-Verlängerung bedingen. Zudem gibt es viele Faktoren, die für eine Verlänge- 


\begin{tabular}{|c|c|c|}
\hline Gruppe & Nierenfunktionsabhängig & Nierenfunktionsunabhängig \\
\hline Analgetika & $\begin{array}{l}\text { Morphin (M6-Glucuronid), Pethi- } \\
\text { din (Norpethidin) }\end{array}$ & Fentanyl, Levomethadon \\
\hline Antibiotika & Ciprofloxacin, Levofloxacin & Moxifloxacin \\
\hline Antiarrhythmika & Sotalol & Amiodaron \\
\hline Antidiabetika & $\begin{array}{l}\text { Glibenclamid, Glimepirid (Hydro- } \\
\text { xymetabolit) }\end{array}$ & Moxifloxacin \\
\hline- & Nateglinid & Pioglitazon \\
\hline Antihypertensiva & Atenolol & - \\
\hline Antikonvulsiva & $\begin{array}{l}\text { Gabapentin, Pregabalin, Lamotri- } \\
\text { gin, Levetiracetam }\end{array}$ & Carbamazepin, Valproat \\
\hline Cholesterinsenker & Bezafibrat, Fenofibrat & Simvastatin, Niazin \\
\hline $\begin{array}{l}\text { Gicht- und Rheumamit- } \\
\text { tel }\end{array}$ & Methotrexat & Hydroxychloroquin, Leflunomid \\
\hline Herz-Kreislauf-Mittel & Digoxin & Digitoxin \\
\hline Psychopharmaka & Lithium, Mirtazapin & $\begin{array}{l}\text { Amitriptylin, Citalopram, Halope- } \\
\text { ridol, Risperidon }\end{array}$ \\
\hline Virostatika & Aciclovir & Brivudin \\
\hline
\end{tabular}

rung der QT-Zeit prädisponieren wie beispielsweise fortgeschrittenes Alter, weibliches Geschlecht, linksventrikuläre Herzinsuffizienz, Diabetes mellitus, Bluthochdruck, Hyperthyreose und Elektrolytstörungen (Hypokaliämie und Hypomagnesiämie) [10]. Allerdings ist eine der häufigsten Ursachen für eine erworbene QT-Zeit-Verlängerung die Einnahme von Medikamenten, die eine Blockierung der schnellen Komponente des verzögerten Gleichrichter-KaliumKanals bewirken und somit zu malignen Herzrhythmusstörungen wie Torsade de pointes führen können [11].

Die pharmakodynamische Wechselwirkung beruht dabei unter anderem auf einem synergistischen Effekt von Medikamenten wie Chloroquin und Hydroxychloroquin und ohnehin QTZeit-verlängernden Medikamenten wie den Makrolidantibiotika (Clarithromycin und Erythromycin), Fluorchinolonen, Cotrimoxazol, Azolantimykotika, Amiodaron, BRAF-Inhibitoren (Dabrafenib und Vemurafenib), SerotoninReuptake-Inhibitoren und trizyklischen Antidepressiva (Online-Abb. 7).

Ein pharmakokinetischer Effekt kann auftreten, wenn die Plasma- und Gewebekonzentrationen eines QT-Zeit-verlängernden Medikaments erhöht sind, beispielswiese durch eine reduzierte $\mathrm{Me}$ tabolisierung der Medikamente (s. CYP450-Inhibitoren, • Abb. 1).

\section{Risikoreiche Medikamenten- gruppen}

Einige Medikamentengruppen gelten als besonders risikoreich. Neben der Gefahr von zum Teil schwerwiegenden Nebenwirkungen kann es zu zahlreichen pharmakodynamischen wie auch -kinetischen Medikamenteninteraktionen kommen.

\section{Orale Retinoide}

Bei der Therapie mit oralen Retinoiden müssen mehrere unerwünschte Interaktionen bedacht werden. Die gleichzeitige Gabe von häufig in der dermatologischen Praxis insbesondere zur Therapie der mittelschweren bis schweren Acne vulgaris verordneten oralen Retinoiden mit Tetrazyklinen erhöht das Risiko einer intrakraniellen Druckerhöhung im Sinne eines Pseudotumors cerebri und stellt somit eine absolute Kontraindikation dar.

Des Weiteren muss unter der Therapie mit systemischen Retinoiden wie Isotretinoin, Alitretinoin, Acitretin oder Bexaroten auf das seltene, aber potenzielle Risiko einer lebensbedrohlichen Rhabdomyolyse geachtet werden [12]. Dieses Risiko ist insbesondere durch gleichzeitige Gabe lipidsenkender Medikamente wie Statine und Fibrate erhöht, die oftmals aufgrund des Anstiegs der Lipide, insbesondere der Triglyzeride, unter einer Retinoidtherapie erfolgt (Online-Abb. 8). Aufgrund dessen müssen bei einer Therapie mit Retinoiden neben Leber- und Lipidwerten auch die Muskelenzyme (CK) regelmäßig laborchemisch kontrolliert werden. Die Patienten sollten darauf hingewiesen werden, starke körperliche Belastungen (beispielsweise Leistungssport) unter der Therapie zu vermeiden und sich bei Auftreten von Muskelschmerzen umgehend bei ihrem behandelnden Arzt vorzustellen (Online-Abb. 9).

\section{Azole}

Das hohe pharmakokinetische Interaktionspotenzial der Azolantimykotika (Fluconazol, Isavuconazol, Itraconazol, Posaconazol und Voriconazol) ist v. a. durch die Verstoffwechselung über das Isoenzym Cytochrom P450 bedingt [14]. Kommen Azole in einer Kombinationstherapie zur Anwendung, ist die Verwendung von Interaktionsprogrammen unerlässlich.

\section{Immunsuppressiva}

Besondere Achtsamkeit ist bei der Anwendung systemischer Immunsuppressiva angebracht, da durch Medikamenteninteraktionen verursachte erhöhte Plasmaspiegel $\mathrm{zu}$ einer gesteigerten Toxizität und einer Suppression des Knochenmarks führen können mit daraus resultierenden lebensbedrohlichen Komplikationen, bedingt durch eine Panzytopenie und potenzielle infektiöse Komplikationen. Unter einer Therapie mit Methotrexat (MTX) sollte insbesondere auf eine gesteigerte Lebertoxizität und die Suppression des Knochenmarks geachtet werden.

Nichtsteroidale Antirheumatika (NSAR), die für gewöhnlich auch zur Behandlung akuter oder chronischer Schmerzen wie bei der Psoriasisarthritis eingesetzt werden, erhöhen die Serumkonzentration von MTX und damit unter anderem das Risiko für aplastische Anämien, Knochenmarksuppression und gastrointestinale Toxizität. Eine engmaschige Kontrolle der Laborwerte sowie die Sensibilisierung des Patienten für das mögliche Auftreten unerwünschter 
Tab. 2 Ausgewählte interaktionsreiche dermatologische Systemtherapeutika

\begin{tabular}{|c|c|c|c|}
\hline Gruppe & Substanzen & Interaktionen & Komplikationen \\
\hline \multirow[t]{2}{*}{ Antimykotika } & Itraconazol & \multirow[t]{2}{*}{ Phenprocoumon } & \multirow[t]{2}{*}{ Erhöhtes Blutungsrisiko } \\
\hline & Ketoconazol & & \\
\hline \multirow[t]{3}{*}{ Antibiotika } & Ciprofloxacin & \multirow[t]{3}{*}{ Phenprocoumon } & \multirow[t]{3}{*}{ Erhöhtes Blutungsrisiko } \\
\hline & Clindamycin & & \\
\hline & Tetrazyklin & & \\
\hline \multirow[t]{2}{*}{ Antikonvulsiva } & Gabapentin & \multirow[t]{2}{*}{ Morphin } & \multirow[t]{2}{*}{ Somnolenz } \\
\hline & Pregabalin & & \\
\hline $\begin{array}{l}\text { Gicht- und Rheu- } \\
\text { mamittel }\end{array}$ & Methotrexat & Ibuprofen & Thrombembolische Komplikationen \\
\hline Glukokortikoide & Prednisolon & NSAR & $\begin{array}{l}\text { Gastrointestinale Blutung, Magenul- } \\
\text { zera }\end{array}$ \\
\hline \multirow[t]{2}{*}{ Immunsuppressiva } & Azathioprin & Allopurinol & $\begin{array}{l}\text { Anämie, Leukopenie, Leberwerter- } \\
\text { höhung }\end{array}$ \\
\hline & Ciclosporin & Statine & Rhabdomyolyse \\
\hline \multirow[t]{6}{*}{ Malariamittel } & \multirow{6}{*}{$\begin{array}{l}\text { Hydroxychlo- } \\
\text { roquin }\end{array}$} & Amiodaron & \multirow[t]{6}{*}{ QT-Intervall-Verlängerung } \\
\hline & & Sotalol & \\
\hline & & Fluorchinolone & \\
\hline & & Makrolide & \\
\hline & & Dabrafenib & \\
\hline & & Vemurafenib & \\
\hline \multirow[t]{4}{*}{ Retinoide } & Isotretinoin & Statine & \multirow[t]{3}{*}{ Rhabdomyolyse } \\
\hline & Acitretin & \multirow[t]{2}{*}{ Fibrate } & \\
\hline & Alitretinoin & & \\
\hline & Bexaroten & Tetrazyklin & Pseudotumor cerebri \\
\hline \multirow[t]{5}{*}{ Virostatika } & \multirow[t]{5}{*}{ Aciclovir } & Ciclosporin & \multirow[t]{5}{*}{ Niereninsuffizienz } \\
\hline & & Aminoglykoside & \\
\hline & & NSAR & \\
\hline & & Penicilline & \\
\hline & & Cephalosporine & \\
\hline
\end{tabular}

Ereignisse sollten bei gleichzeitiger Gabe von MTX und NSAR erfolgen (OnlineAbb. 10).

Unter einer Ciclosporin-Therapie sind aufgrund der geringen therapeutischen Breite des Medikaments sowie der durch CYP-Induktoren und -Inhibitoren beeinflussten Metabolisierung (s. Abb. 1) regelmäßige Plasmaspiegelbestimmungen zur Verhinderung toxischer Nebenwirkungen sinnvoll. Insbesondere die gleichzeitige Gabe anderer nephrotoxischer, zytotoxischer oder immunsuppressiver Arzneimittel erfordert ein genaues Monitoring und ggf. eine Anpassung der Ciclosporin-Dosis.

Protonenpumpeninhibitoren (PPI), die in großem Umfang und oftmals über einen langen Zeitraum für die Behandlung von Magensäure-beding- ten Erkrankungen eingesetzt werden, erhöhen das Risiko für klinisch signifikante Arzneimittelinteraktionen bei Patienten, die gleichzeitig weitere Arzneimittel einnehmen. Die Verabreichung von PPI erhöht den intragastrischen $\mathrm{pH}$ Wert, wodurch die Hydrolyse von Mycophenolat-Mofetil verlangsamt wird, was wiederum zu einer verringerten $\mathrm{Re}$ sorption und maximalen Verfügbarkeit von Mycophenolsäure führt (OnlineAbb. 11).

$\mathrm{Zu}$ den primär bei therapierefraktärer Psoriasis angewandten Biologika (TNF[Tumor-Nekrose-Faktor]- $\alpha$ Inhibitoren und IL[Interleukin]-12/23Inhibitoren) gibt es bisher wenige Informationen über beschriebene Arzneimittelinteraktionen. Dennoch wird zur Vermeidung des theoretischen Risikos einer additiven Immunsuppression sowie einer infektiösen Komplikation zur Vorsicht bei der Kombination mit anderen immunsuppressiven Medikamenten geraten.

Impfungen mit Lebendimpfstoffen sollten unter immunsuppressiver Therapie zurückgestellt werden und frühestens 3 Monate nach Absetzten des Immunsuppressivums gegeben werden. Auch die Wirksamkeit anderer Impfungen kann unter Umständen unter der immunsuppressiven Therapie verringert sein.

Ein erhöhtes Risiko für die Entstehung von nichtmelanozytärem Hautkrebs (NMSC) zählt zum Nebenwirkungsprofil von zahlreichen Immunsuppressiva wie Ciclosporin und Azathioprin. Pharmakoepidemiologische Studien haben jüngst auch für die Exposition mit steigenden kumulativen Dosen von Hydrochlorothiazid ein erhöhtes Risiko für die Entstehung von nichtmelanozytärem Hautkrebs gezeigt [13]. Bei Patienten unter immunsuppressiver Therapie sollte aus dermatologischer Sicht die Kombination von immunsupprimierenden Medikamenten mit anderen NMSC entstehungsfördernden Medikamenten gemieden werden. Im Hinblick auf eine gleichzeitige antidiuretische Therapie mit Hydrochlorothiazid sollte eine vorsichtige Risiko-Nutzen-Abwägung erfolgen und ggf. auf alternative Medikamente umgestellt werden.

\section{Fazit für die Praxis}

- Arzneimittelkombinationen sind häufig und müssen kritisch wahrgenommen werden (Online-Abb. 12).

- Mit der Anzahl gleichzeitig eingenommener Arzneimittel steigt auch das Potenzial für unerwünschte Wechselwirkungen - insbesondere in höherem Lebensalter und bei chronischen Erkrankungen (• Tab. 2).

- Kenntnisse über die wichtigsten Prinzipien pharmakokinetischer und pharmakodynamischer Arzneimittelinteraktionen sind daher von besonderer Bedeutung. In diesem Zusammenhang ist insbesondere die Metabolisierung über das Cytochrom-P450-System wichtig, da 


zahlreiche Arzneimittel überwiegend
über diese Enzyme verstoffwechselt
werden.
Klinische Konsequenzen zur Ver-
meidung unerwünschter und teils
lebensbedrohlicher Wechselwir-
kungen sind je nach Arzneimittel-
kombinationen eine entsprechende
Dosisanpassung, eine zeitlich ver-
setzte Einnahme der Medikamente
oder auch ein Wechsel bzw. Absetzen
von Wirkstoffen.
Programme zur Überprüfung von
Medikamenteninteraktionen sind im
klinischen Alltag unverzichtbar.

\section{Korrespondenzadresse}

\section{Dr. med. Kristina Krause}

Klinik für Dermatologie, Universitätsklinikum Düsseldorf

Moorenstr. 5, 40225 Düsseldorf, Deutschland kristina.krause@med.uni-duesseldorf.de

Funding. Open Access funding enabled and organized by Projekt DEAL.

\section{Einhaltung ethischer Richtlinien}

Interessenkonflikt. K. Krause, K. Jahn und B. Homey geben an, dass kein Interessenkonflikt besteht.

Für diesen Beitrag wurden von den Autoren keine Studien an Menschen oder Tieren durchgeführt. Für die aufgeführten Studien gelten die jeweils dort angegebenen ethischen Richtlinien.

Open Access. Dieser Artikel wird unter der Creative Commons Namensnennung 4.0 International Lizenz veröffentlicht, welche die Nutzung, Vervielfältigung, Bearbeitung, Verbreitung und Wiedergabe in jeglichem Medium und Format erlaubt, sofern Sie den/die ursprünglichen Autor(en) und die Quelle ordnungsgemäß nennen, einen Link zur Creative Commons Lizenz beifügen und angeben, ob Änderungen vorgenommen wurden.

Die in diesem Artikel enthaltenen Bilder und sonstiges Drittmaterial unterliegen ebenfalls der genannten Creative Commons Lizenz, sofern sich aus der Abbildungslegende nichts anderes ergibt. Sofern das betreffende Material nicht unter der genannten Creative Commons Lizenz steht und die betreffende Handlung nicht nach gesetzlichen Vorschriften erlaubt ist, ist für die oben aufgeführten Weiterverwendungen des Materials die Einwilligung des jeweiligen Rechteinhabers einzuholen.

Weitere Details zur Lizenz entnehmen Sie bitte der Lizenzinformation auf http://creativecommons.org/ licenses/by/4.0/deed.de.

\section{Literatur}

1. Alhawassi TM, Krass I, Bajorek BV, Pont LG (2014) A systematicreview of the prevalence and riskfactors for adverse drug reactions in the elderly in the acute care setting. Clin Interv Aging 9:2079-2086

2. Gallagher PF, Barry PJ, Ryan C, Hartigan I, O'Mahony D (2008) Inappropriate prescribing in an acutely ill population of elderly patients as determined by Beers' criteria. Age Ageing 37:96-101

3. Zabost A, Brzezińska S, Kozińska M et al (2013) Correlation of $\mathrm{N}$-acetyltransferase 2 genotype with isoniazid acetylation in Polish tuberculosis patients. Biomed Res Int. https://doi.org/10.1155/ 2013/853602

4. Cascorbi I (2012) Drug interactions-principles, examples and clinical consequences. Dtsch Arztebl Int 109:33-34

5. Verbeurgt P, Mamiya T, Oesterheld J (2014) How common are drug and gene interactions? Prevalence in a sample of 1143 patients with CYP2C9, CYP2C19 and CYP2D6 genotyping. Pharmacogenomics 15:655-665

6. Seden K, Dickinson L, Khoo S, Back D (2010) Grapefruit-drug interactions. Drugs 70:2373-2407

7. Aliud Pharma (2020) Fachinformation Methotrexat AL $25 \mathrm{mg} / \mathrm{ml}$. http://fachinformation.srz.de/pdf/ aliudpharma/methotrexatal25mgmlinjektionsl \%C3\%B6sunginfertigspritze.pdf. Zugegriffen: 30 . März 2020

8. Hartmann B, Czock D, Keller F (2010) Arzneimitteltherapie bei Patienten mit chronischem Nierenversagen. Dtsch Arztebl Int 107(37):647-656. https:// doi.org/10.3238/arztebl.2010.0647

9. Al-Quteimat OM, Al-Badaineh MA (2013) Methotrexate and trimethoprim-sulphamethoxazole: extremely serious and life-threatening combination. JClin Pharm Ther 38:203-205

10. Al-Khatib SM, LaPointe NMA, Kramer JM, Califf RM (2003) What clinicians should know about the QT interval. J Am Med Assoc 289:2120-2127

11. van Noord C, Eijgelsheim M, Stricker BHC (2010) Drug- and non-drug-associated QT interval prolongation. Br JClin Pharmacol 70:16-23

12. Hartung B, Merk HF, Huckenbeck W et al (2012) Severe generalised rhabdomyolysis with fatal outcome associated with isotretinoin. Int J Legal Med 126:953-956

13. Pedersen SA, Arnspang S (2018) Hydrochlorothiazide use and risk of nonmelanoma skin cancer: a nationwide case-control study from Denmark. J Am Acad Dermatol 78:673-681

14. Petri H (2017) Arzneimitteltherapiesicherheit: Interaktionspotenzial der Azol-Antimykotika. Dtsch Arztebl Int 22:114-117. https://doi.org/10. 3238/PersInfek.2017.04.28.08

15. Arakawa Yetal (2018) Renal clearance and intracellular half-life essentially determine methotrexate toxicity: a case series. JAAD Case Rep 5(1):98-100. https://doi.org/10.1016/j.jdcr.2018.10.022

16. Ohls RK, Christensen RK (2003) Chapter 20. Diseases of the blood. In: Behrman RE, Kliegman RM, Jenson HB (Hrsg) Nelson textbook of pediatrics, 17. Aufl. Saunders, Philadelphia

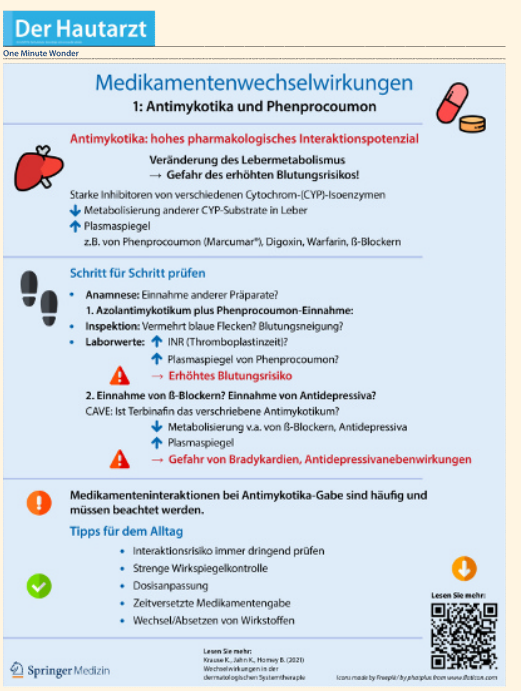

\section{Zum One Minute Wonder dieser Ausgabe}

Freuen Sie sich ab Ausgabe 1/2021 auf das One Minute Wonder, Ihr monatliches kurzes Fachwissen to go (originell - markant wissenswert).

Den Anfang macht ein OMW zu Medikamentenwechselwirkungen.

Teil 1: Antimykotika und Phenprocoumon

One Minute Wonder zum Beitrag: Krause K, Jahn K, Homey B (2021) Wechselwirkungen in der dermatologischen Systemtherapie. Hautarzt 72: 44-49, www.springermedizin.de/link/18629268

Erstellt im November 2020 von:

Dr. J. Kind, Heidelberg

Beratung: PD Dr. Dr. A. Zink, München Geprüft und freigegeben im November 2020 von: Dr. K. Krause, Düsseldorf, und PD Dr. Dr. A. Zink, München

Gestaltung: E. Migur, Le-Tex Leipzig

Haben Sie Fragen oder Anregungen zu diesem One Minute Wonder?

Vielleicht haben Sie Erfahrungen gesammelt, inwieweit eine ausgedehnte topische Therapie mit Antimykotika bei großfächigen Dermatomykosen den CYP-Stoffwechsel beeinflusst?

Die Redaktion freut sich auf regen Austausch (julie.kind@springer.com). 